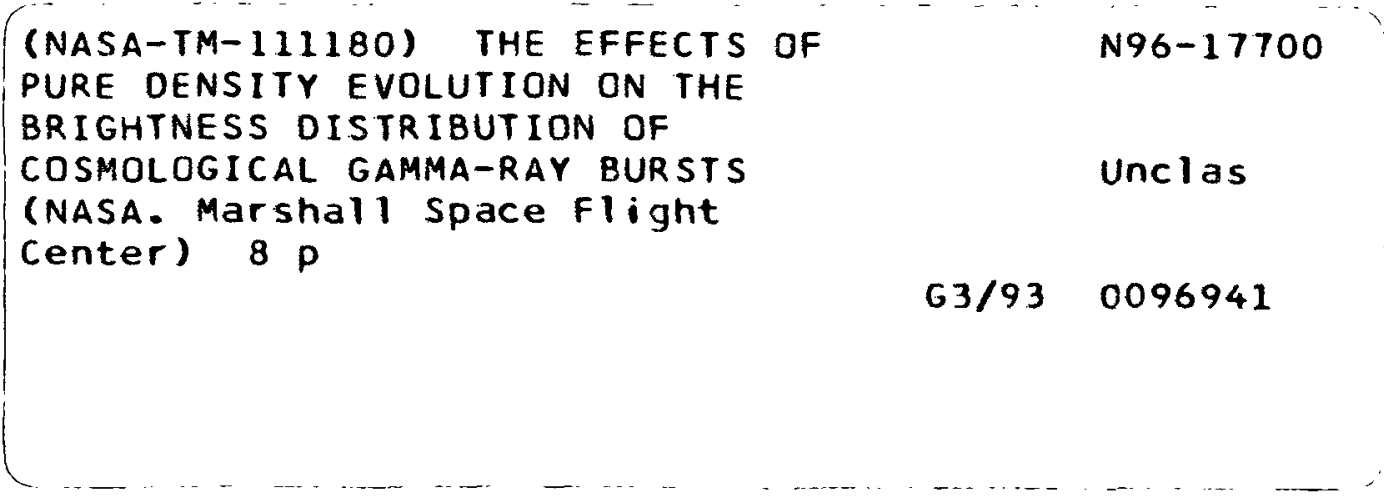




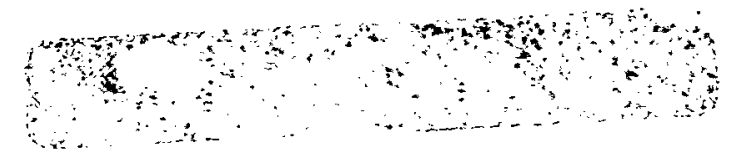

THE ASTROPHYSICAL JOURNAL, 447:474-481, 1995 July 10 (C) 1995. The American Astronomical Society. All rights reserved. Printed in U.S.A.

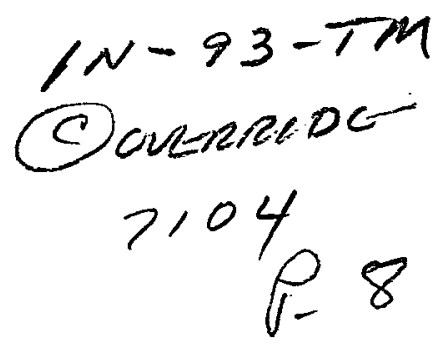

\title{
THE EFFECTS OF PURE DENSITY EVOLUTION ON THE BRIGHTNESS DISTRIBUTION OF COSMOLOGICAL GAMMA-RAY BURSTS
}

\author{
J. M. HoraCk, ${ }^{1}$ A. G. EMSLIE, ${ }^{2}$ aNd D. H. HartmanN ${ }^{3}$ \\ Received 1994 June 27; accepted 1995 January 20
}

\begin{abstract}
.
In this work, we explore the effects of burst rate density evolution on the observed brightness distribution of cosmological gamma-ray bursts. Although the brightness distribution of gamma-ray bursts observed by the BATSE experiment has been shown to be consistent with a nonevolving source population observed to redshifts of order unity, evolution of some form is likely to be present in the gamma-ray bursts. Additionally, nonevolving models place significant constraints on the range of observed burst luminosities, which are relaxed if evolution of the burst population is present. In this paper, three analytic forms of density evolution are examined. In general, forms of evolution with densities that increase monotonically with redshift require that the BATSE data correspond to bursts at larger redshifts, or to incorporate a wider range of burst luminosities, or both. Independent estimates of the maximum observed redshift in the BATSE data and/or the range of luminosity from which a large fraction of the observed bursts are drawn therefore allow for constraints to be placed on the amount of evolution that may be present in the burst population. Specifically, if recent measurements obtained from analysis of the BATSE duration distribution of the actual limiting redshift in the BATSE data at $z_{\mathrm{lim}}=2$ are correct, the BATSE $N(P)$ distribution in a $\Lambda=0$ universe is inconsistent at a level of $\sim 3 \sigma$ with nonevolving gamma-ray bursts and some form of evolution in the population is required. The sense of this required source evolution is to provide a higher density, larger luminosities, or both with increasing redshift.
\end{abstract}

Subject headings: cosmology: theory - gamma rays: bursts

\section{INTRODUCTION}

The paradigm of a cosmological origin for gamma-ray bursts has gained greater acceptance by the scientific community in light of the Burst and Transient Source Experiment (BATSE) results which indicate that the gamma-ray bursts are distributed isotropically on the sky, yet possess an integral brightness distribution that deviates significantly from the $-3 / 2$ power law indicative of homogeneity in Euclidean space (Meegan et al. 1992). Numerous models of cosmological burst production have been postulated, including NS-NS mergers e.g., Piran et al. (1991), emission from massive black holes in the center of galaxies (Brainerd 1994), and failed supernovae (Woosley 1993). Through a variety of analysis techniques, Wickramasinghe et al. (1993), Mao \& Paczyński (1992), Piran (1992), Fenimore et al. (1993), and others have shown that the observed brightness distribution of gamma-ray bursts, $\mathscr{N}(>P)$, is consistent with a population of mono-luminous sources distributed with a constant comoving rate density out to a limiting redshift of $z_{\max } \sim 1$ in a Friedmann universe with cosmological constant $\Lambda=0$.

Emslie \& Horack (1994) have relaxed the restriction of mono-luminosity and also allow a general value for the cosmological constant $\Lambda$ in their analysis of the BATSE brightness distribution. Utilizing an integral moment analysis technique, they find not only that the limiting redshift for most cosmological scenarios is indeed of order unity, but also that the gamma-ray burst luminosity function must be fairly narrow for such scenarios, with most observed bursts originating from a

\footnotetext{
' NASA/Marshall Space Flight Center, ES-84, Huntsville, AL 35812.

2 Department of Physics, University of Alabama in Huntsville, Huntsville, AL 35899.

${ }^{3}$ Department of Physics \& Astronomy, Clemson University, Clemson, SC 29634.
}

luminosity range that is no wider than a factor of $\sim 10$. Emslie and Horack additionally show that if one assumes the observed luminosity function is substantially wider than this value, the observed BATSE brightness distribution is consistent with a constant comoving number density only in the context of an accelerating universe with a positive value for $\Lambda$.

None of these previously mentioned analyses examine in detail the occurrence of evolution in either the comoving source density function $n_{c}$ or in the luminosity function $\phi(L)$. Evolution, either of the form $n_{c}(z), \phi(L, z)$, or both may be present at some level. It is therefore important to explore the effects of evolution on the observed GRB brightness distribution and to determine the consistency of various evolutionary scenarios with the observed $N(P)$ distribution.

In this work, we utilize the expanded dataset offered by the second BATSE gamma-ray burst catalog (Meegan et al. 1994). We continue to assume that the luminosity function $\phi(L)$ is independent of $z$, regardless of its particular form, but we do allow the comoving rate number density of gamma-ray bursts to be a function of redshift $z$ [or some equivalent parameter such as cosmic look-back time $\tau(z)]$. This mode of evolution is known as pure density evolution (PDE), and has been adopted for several reasons. First, because we are only beginning to explore the effects of source evolution in the context of cosmological models, it makes sense to begin with the simplest set of assumptions and grow into more complex evolutionary scenarios only if necessary. PDE is the simplest mathematical representation of evolution, where all evolutionary effects are contained in the comoving density function $n_{c}(z)$. Second, because the luminosity function is independent of redshift, the effects of $n_{c}(z)$ and $\phi(L)$ are separable and hence the methodology of Emslie \& Horack (1994), based on the technique of integral moment analyses, can be applied to the observed brightness distribution to determine the range of cosmological 
scenarios that are compatible with the data. [Redshift-dependent luminosity functions that are separable, i.e., $\phi(L, z)=\phi(L) g(z)$, can also be analyzed under this methodology.] Third, PDE may indeed accurately describe the evolutionary situation for a number of cosmological burst models, for example the NS-NS merger scenario where the burst's luminosity depends only on $z$-independent physical quantities such as the mass of a neutron star, but the event rate would depend on redshift because the formation of progenitor systems traces galactic star formation which is likely to vary in time (e.g., Hartmann 1995).

We examine the compatibility between three different evolutionary scenarios and the brightness distribution of the second BATSE catalog of data (Meegan et al. 1994):

1. Power-law evolution, where the comoving rate density of bursts is proportional to $(1+z)^{\beta}$, where $\beta$ is a parameter.

2. Exponential evolution, where $n_{c}(z)$ is proportional to exp $\left(\eta \tau H_{0}\right)$, where $\tau$ is the cosmic look-back time, $H_{0}$ is the Hubble constant, and $\eta$ is a variable scaling parameter.

3. Quasar-like density evolution, where the burst rate density is proportional to $\exp \left[-\left(z-z_{\text {peak }}\right)^{2} / 2 \sigma^{2}\right]$, i.e., centered on a redshift $z_{\text {peak }}$ with a spread of $\sigma$.

Of course one has an infinite number of a priori choices for $n_{c}(z)$, however we choose these three for their simplicity so that the effects of PDE can be more easily understood in the absence of many free parameters.

In the following section, we describe the analysis technique used for each of these three evolutionary scenarios. Subsequent sections of the paper discuss results from each of these three scenarios in detail. In the final section of the paper, we discuss conclusions regarding the modes of PDE compatible with the BATSE data.

\section{ANALYSIS TECHNIQUE}

The methodology by which compatibility of a given evolutionary scenario is determined in this work is identical to the analysis of Emslie \& Horack (1994). For a cosmological burst distribution, the normalized integral moments $\left\{N^{(m)}\right\}$ of the differential BATSE brightness distribution $N(P)$ can be expressed as (see Horack \& Emslie 1994)

$$
\left\{N^{(m)}\right\}=\left\{\Phi_{*}^{(m)}\right\} \frac{\int_{0}^{z_{\max }} n_{c}(z) f(z) r(z)^{2-2 m}(1+z)^{-\alpha m-1} d z}{\int_{0}^{z_{\max }} n_{c}(z)(1+z)^{-1} f(z) r(z)^{2} d z},
$$

where

$$
f(z)=\frac{1}{\left[2 \sigma_{0}(1+z)^{3}+\left(1+q_{0}-3 \sigma_{0}\right)(1+z)^{2}+\left(\sigma_{0}-q_{0}\right)\right]^{1 / 2}} .
$$

Here, $\sigma_{0}$ is the density parameter, $q_{0}$ is the deceleration parameter, and $\alpha$ is the burst power-law photon spectral index, which we take to be a representative value of 2 throughout this analysis. The $\left\{\Phi_{*}^{(m)}\right\}$ are the normalized integral moments of the function $\phi_{*}(L)$ which represents the number of bursts with peak luminosities between $L$ and $L+d L$ that are accessible to the BATSE detector, and also incorporates various normalizations such as the current value of the scale-factor $S_{0}$ and the Hubble constant $H_{0}$. Although the relationship between $\phi_{*}(L)$ and $\phi(L)$, the luminosity distribution of all bursts, depends on the form of $n_{c}(z)$, for the purposes of this analysis, one only needs to assert that the function $\phi_{*}(L)$ exists and that it is non-negative everywhere.

The integral moments of the BATSE differential $N(P)$ distribution, along with their associated uncertainties, are easily measured from the observational data and are represented on the left-hand side of equation (1). Horack \& Emslie (1994) provide a thorough discussion of how these numbers are obtained. Briefly, the $m$ th integral moment $\left\{N^{(m)}\right\}$ is simply computed as

$$
\left\{N^{(m)}\right\} \equiv\left\langle P^{m}\right\rangle,
$$

where $P$ is the $0.256 \mathrm{~ms}$ peak flux of gamma-ray bursts in the energy range $50-300 \mathrm{keV}$. Only those bursts with peak fluxes in excess of 0.5 photons $\mathrm{cm}^{-2} \mathrm{~s}^{-1}$ are utilized, to avoid the instrumental selection effects that are known to bias the intensity distribution below this value (Fishman et al. 1994). Uncertainties in these quantities, like those in the computation of the angular distribution moments $\langle\cos \theta\rangle$ and $\left\langle\sin ^{2} b\right\rangle$, are due to both uncertainties in the peak flux $P$ of each individual gamma-ray burst, as well as statistical uncertainties due to the finite number of bursts in the sample. Horack et al. (1994a), Briggs et al. (1994), and others have shown that in computing the angular distribution moments, the total uncertainty in the measured value is dominated by the $1 / \sqrt{N}$ statistical uncertainties due to the finite number of bursts. Not surprisingly, this is also the case with the brightness distribution moments $\left\{N^{(m)}\right\}$ (Horack \& Emslie 1994; Pendleton et al. 1995).

After computation of the left-hand side of equation (1) from the data, if one subsequently assumes a cosmological model $\left(\sigma_{0}, \dot{q}_{0}\right)$ and a burst distribution $n_{c}(z)$ out to a limiting redshift $z_{\max }$, the integral on the right-hand side of equation (1) can be computed and the set of numbers $\left\{\Phi_{*}^{(m)}\right\}$ and their uncertainties can be derived. If the assumed cosmological scenario is valid, these numbers are the integral moments of the associated $\phi_{*}(L)$ function, from which minimal information on $\phi_{*}(L)$ and, since $n_{c}(z)$ has been assumed for the given model, $\phi(L)$ can be deduced. Whether or not this information is accurate depends on the validity of the assumed cosmological scenario.

It might seem that, with no a priori information on either $\phi(L)$ or $n_{c}(z)$, little information can in fact be obtained through such methods. However, the integral moments of a given function are not all independent quantities. For example, for a function $f(r)$, the second moment $\left\langle r^{2}\right\rangle$ must be larger than (or equal to) the square of the first moment $\langle r\rangle^{2}$, in order that $f(r)$ have a realizable (non-negative) variance. Consequently, an arbitrary set of computed numbers need not be valid moments for the $\left\{\Phi_{*}^{(m)}\right\}$. In order that a given set of numbers represent the moments of a positive semi-definite function, these numbers must satisfy conditions known as the Wald inequalities (Wald 1939). These inequalities state that a set of numbers $\mu_{r}, \mu_{s}, \mu_{t}$ can be realized as moments of order $r, s, t(r<s<t)$ of some non-negative distribution function defined on the interval $[0, \infty)$ if and only if

$$
\mu_{r} \geq 0 ; \quad \mu_{s} \geq \mu_{r}^{s / r} ; \quad \mu_{r}^{t} \mu_{s}^{r} \mu_{t}^{s} \geq \mu_{r}^{s} \mu_{s}^{t} \mu_{r}^{r} .
$$

For the problem at hand, then, if a set of numbers $\left\{\Phi_{*}^{(m)}\right\}$ are obtained through the assumption of a cosmological model and employment of the BATSE $N(P)$ normalized integral moments $\left\{N^{(m)}\right\}$, and these derived numbers violate any of the inequalities in equation (4), not only can these numbers not be the moments of the $\phi_{*}(L)$ function, they cannot be the moments of 
any function. One is therefore forced to conclude that the cosmological model and evolutionary scenario that was chosen to generate these numbers are incompatible with the BATSE data for all $\phi(L)$ at the appropriate level of significance indicated by the propagated uncertainties. In this manner, the viability of certain cosmological evolutionary models can be ruled out.

Specifically for this analysis, we have chosen those moments from the observed $N(P)$ distribution which can be measured to an accuracy of $\sim 10 \%-15 \%$ or better, corresponding to values of $m=-1,-2, \ldots,-6$. We then select values in the $\left(\sigma_{0}, q_{0}\right)$ plane, combined with an assumed form for $n_{c}(z)$, and use the wald inequalities to determine the largest limiting redshift $z_{\max }$ for which a mathematically tenable $\phi_{*}(L)$ function can be constructed. This generates a volume of parameter space in which all viable cosmological scenarios with the assumed comoving density function must exist. Figure 1 shows this volume as obtained by the method of Emslie \& Horack (1994) for a nonevolving comoving density $n_{c}(z)=$ constant. Emslie and Horack also have computed surfaces corresponding to the +1 $\sigma$ and $+3 \sigma$ uncertainties applied to the observed moments $\left\{N^{(m)}\right\}$. The basic form of these surfaces is the same.

In addition to constraining the possible cosmological models consistent with the BATSE data, further information may be obtained by realizing that the upper surface to this parameter volume consists of cosmological models which admit luminosity functions $\phi_{*}(L)$ which are just compatible with the Wald inequalities, i.e., $\approx \delta\left(L-L_{0}\right)$. The height of the upper surface therefore corresponds to the maximum redshift obtained from the BATSE data for a given $\left[n_{c}(z), \sigma_{0}, q_{0}\right]$ assumption with a burst population that is mono-luminous. The farther a model lies below this limiting surface, the greater the flexibility in choosing a $\phi_{*}(L)$.

For example, if $z_{\max } \ll 1$, the nonevolving bursts are embedded in a Euclidean space and evolutionary effects are negligible. The deviation from a $-3 / 2$ power law in the $\log \mathcal{N}(>P)$ profile is then due to the instrumental truncation of lowluminosity bursts at large distances (which therefore have a brightness below the BATSE threshold), and the rate at which the curve deviates from the $-3 / 2$ power law is a reflection of the shape of the luminosity distribution. Steep $\phi_{*}(L)$ cause a relatively rapid fall-off as is observed, while shallow $\phi_{*}(L)$ would cause a more gradual fall-off in contradiction to the

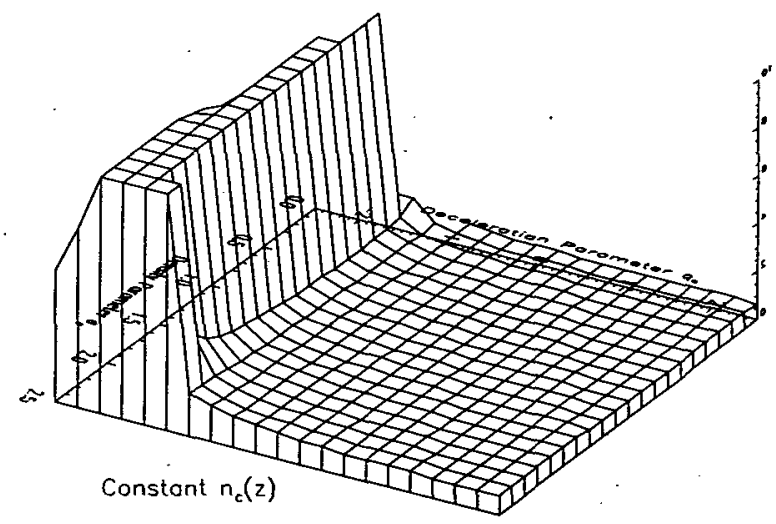

FiG. 1.-Limiting redshift $z_{\max }\left(\sigma_{0}, q_{0}\right)$ derived for a nonevolving population of cosmological gamma-ray bursts. Cosmological scenarios that are compatible with the BATSE data lie on or below the surface. (Emslie \& Horack 1994).
BATSE observations. The restriction on overly shallow forms of $\phi_{*}(L)$ is consistent with the low height of the $z_{\max }$ surface, which does not permit models to lie substantially below it.

Therefore, regions of the parameter volume which prohibit either large values of $z_{\max }$ or which prohibit the model from lying substantially below the upper surface are therefore constrained to have relatively steep and/or narrow observed luminosity ranges (see Horack, Emslie, \& Meegan 1994b; Emslie \& Horack 1994), depending on the form of the radial distribution. As can be seen from Figure 1, this is indeed the case for most nonevolving scenarios with $q_{0}$ larger than zero, including all models for which $\Lambda=0$. We shall see in subsequent sections that evolutionary models can permit a larger value of $z_{\max }$ and hence a shallower form of $\phi_{\star}(L)$.

\section{POWER-LAW EVOLUTION IN REDSHIFT}

The first evolutionary scenario we examine consists of a comoving burst density that evolves as

$$
n_{c}(z) \propto(1+z)^{\beta},
$$

where $\beta$ is a variable index that can be used to increase or decrease the amount and direction of the evolution present in the scenario. Although not necessarily motivated from observational data, this form of evolution is useful in determining what effects are to be found for various amounts of evolution in the density of bursts.

We have determined the largest permissible value of $z_{\max }\left(\sigma_{0}, q_{0}\right)$ for a variety of values $\beta$ ranging from -2 to 2 . The $z_{\max }\left(\sigma_{0}, q_{0}\right), z_{\max }+1 \sigma$, and $z_{\max }+3 \sigma$ surfaces with $\beta=0$ are obviously identical to those of the nonevolving case $(\S 2$, Fig. 1 , Emslie \& Horack 1994). For comparison with a moderately evolving $n_{c}(z)$, Figure 2 shows the derived $z_{\max }\left(\sigma_{0}, q_{0}\right)$ limiting surface for $\beta=1.7$; corresponding to nearly 3.25 times the rate of bursts per unit volume of comoving space at $z=1$ than are present locally. The general shape of the two limiting surfaces in Figure 1 and Figure 2 are the same, however with the increased value of $\beta$, larger values of $z_{\max }$ are admitted and the plateau region adopts a slightly more curved shape.

This result is easily understood when one considers the log $\mathscr{N}(>P)$ versus $\log P$ curve. With an excess of bursts at large redshifts/distances, the curve at the low- $P$ end of the distribution tends to display a steeper slope than would be found for a population with no evolution. In fact, in a Euclidean distribution, the slope would be steeper than the uniform density value

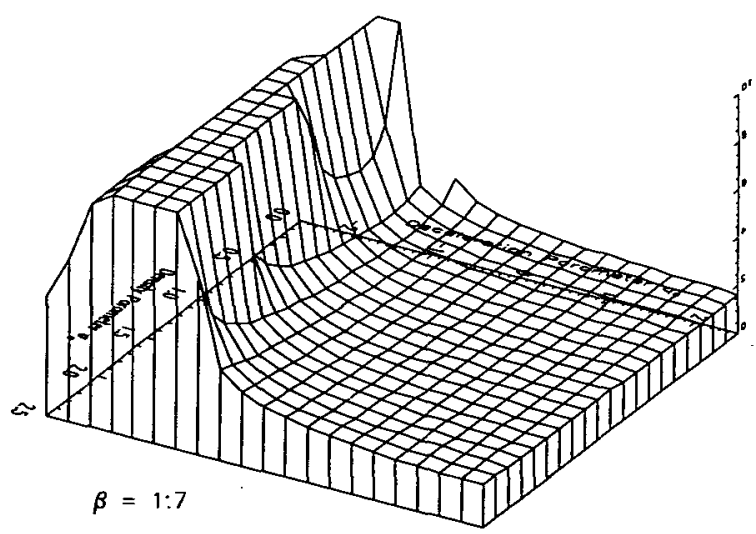

FIG. 2-Limiting redshift $z_{\max }\left(\sigma_{0}, q_{0}\right)$ for bursts evolving as $n_{c}(z) \propto(1$ $+z)^{1.7}$. 
of $-3 / 2$. Larger redshifts are therefore required over the observed range of intensities to introduce stronger cosmological bending of the $\mathscr{N}(>P)$ curve to the flatter (slope $\sim-0.8$ ) form observed in the BATSE data. This requirement of larger $z_{\max }$ is therefore a general one for all evolutionary scenarios which have monotonically increasing $n_{c}(z)$ and a narrow $\phi_{*}(L)$.

Figure 3 shows plots of the maximum limiting redshift $z_{\max }$ determined for three simple cosmological scenarios as a function of the index $\beta$. All three of these scenarios have a zero cosmological constant, with values of the deceleration parameter $q_{0}$ equal to 0 (diamonds), 0.5 (triangles), and 1.0 (squares). Cosmological models with $\Lambda=0$ and power-law density evolution must possess limiting redshifts equal to or less than the value of $z_{\max }$ shown in order to be consistent with the observed BATSE brightness distribution. Furthermore, models with limiting redshifts very near the value of $z_{\max }$ in the figure correspond to nearly mono-luminous bursts.

This figure permits one to draw various conclusions regarding the amount of power-law source evolution that can be present in the burst population depending on the actual limiting redshift in the BATSE burst sample, and some general assumptions regarding the type of luminosity function the observed bursts possess.

As an example, one independent measurement of the limiting redshift of bursts in the BATSE sample may have been given by the work of Norris et al. (1995), who used the duration distributions of bright and dim bursts to search for cosmological time-dilation. It is well-known that the average duration for a set of gamma-ray bursts at redshift $z_{\text {dim }}$, when compared with the average duration from a set of bursts at smaller redshift $z_{\text {bri }}$, can be expressed as

$$
\frac{\left\langle T_{\mathrm{dim}}\right\rangle}{\left\langle T_{\mathrm{brt}}\right\rangle}=\frac{1+z_{\mathrm{dim}}}{1+z_{\mathrm{brt}}} .
$$

Norris et al. have measured this ratio to be slightly in excess of 2. Combined with the assumption that the bright gamma-ray bursts are located at a redshift of $z_{\mathrm{brt}} \approx 0.3$ (Norris et al. 1995), their analysis indicates that the BATSE data display timedilation effects consistent with the dimmest bursts at limiting redshifts of $\sim 2$. A similar time-dilation effect has also been observed by Wijers \& Paczyński (1994). However, Emslie \& Horack (1994) have shown that a limiting redshift of $z_{\max } \approx 2$ is

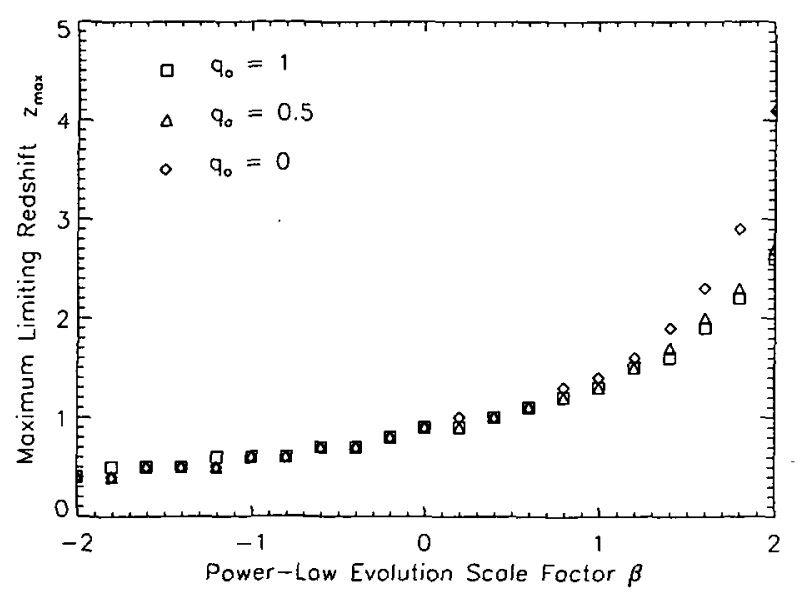

Fig. 3.-Limiting redshift $z_{\max }$ vs. power-law evolution index $\beta$ for three $\Lambda=0$ cosmologies with an evolving burst population $n_{c}(z) \propto(1+z)^{\beta}$. inconsistent with the BATSE data and a nonevolving population of gamma-ray bursts at the $+3 \sigma$ level. Evolution in the source population provides a means of reconciling the apparent limiting redshift obtained with time-dilation measurements and the BATSE $N(P)$ distribution.

If one chooses this value of $z_{\text {lim }}=2$ as an independent measurement of the actual limiting redshift in the BATSE data, evolutionary scenarios that are not inconsistent with the BATSE data at some level can only be found in Figure 3 where the model lies near $z_{\max }=2$, and below the curves displayed in the figure. This restricts the admissible forms of power-law evolution functions to those where $\beta$ is larger than 1.4-1.6, depending only slightly on the chosen value of $q_{0}$. Therefore, if the Norris et al. (1994) limiting redshift of 2 is the correct value for the BATSE data, power-law evolutionary scenarios consistent with the BATSE $N(P)$ distribution and the measured limiting redshift must be at least as strongly increasing as $n_{c}(z) \propto(1$ $+z)^{1.5}$, and negative values of $\beta$ are excluded. This conclusion is furthermore independent of the luminosity function chosen for the bursts.

An additional assumption regarding the range of luminosity present in the BATSE data can constrain the maximum amount of evolution that is permissible. For example, if one additionally assumes that the luminosity function of the observed gamma-ray bursts is rather narrow (an assumption that may be supported in the context of $\Lambda=0$ cosmologies by the rapid turnover in the integral number-intensity distribution observed by BATSE; Emslie \& Horack 1994; Mao \& Paczyński 1993), one further constrains the locus of points in Figure 3 consistent both with the BATSE $N(P)$ distribution and these additional studies. Consistent points now not only must lie very near to the plotted curves in Figure 3. For an absolute redshift limit of $z_{\max } \sim 2$ and standard candle burst sources, the amount of power-law PDE in the comoving density cannot be any more or less severe than about $\beta \approx 1.5-2.0$.

\section{EXPONENTIAL EVOLUTION IN COSMIC LOOK-BACK TIME}

The second form of PDE is exponential, with the comoving density of bursts following the form

$$
n_{c}(z) \propto \exp \left[\eta \tau(z) H_{0}\right],
$$

where $\tau(z)$ is the cosmic look-back time given by

$$
\tau(z)=\frac{1}{H_{0}} \int_{0}^{z} \frac{f\left(z^{\prime}\right)}{\left(1+z^{\prime}\right)} d z^{\prime},
$$

with $H_{0}$ being the Hubble constant, $f(z)$ is given by equation (2), and $\eta$ is an exponential scaling parameter.

The procedure for analysis of this model of evolution is formally identical to the previous section. The $z_{\max }\left(\sigma_{0}, q_{0}\right)$ surface is constructed using the moments of the BATSE $N(P)$ distribution along with the Wald inequalities. For points above the surface, no physical $\phi_{*}(L)$ function can be constructed given the BATSE data and the assumed cosmological parameters. Figure 4 shows two of the $z_{\max }\left(\sigma_{0}, q_{0}\right)$ surfaces for values of $\eta$ equal to 3 and 9 .

Qualitatively, these surfaces are similar in shape to the limiting surface derived with no density evolution (Fig. 1). As was seen in the case of power-law density evolution, as the amount of evolution in the burst population increases ( $\eta$ becomes larger), the maximum limiting redshift also increases to provide 

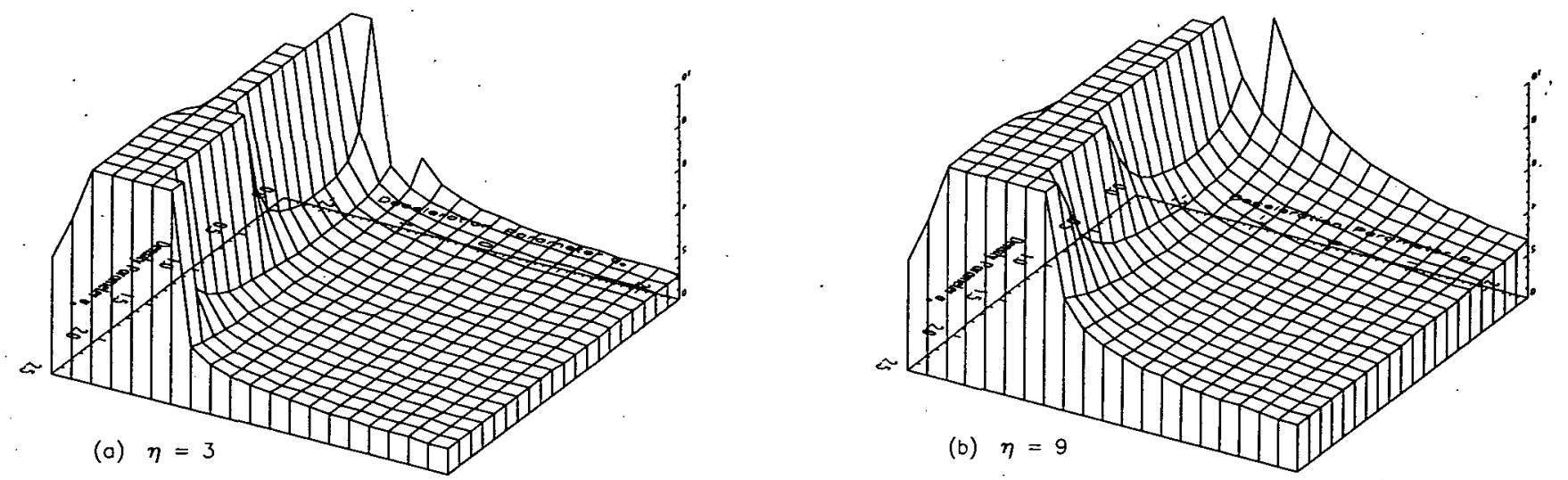

FIG. 4.--Limiting redshift $z_{\max }\left(\sigma_{0}, q_{0}\right)$ surfaces for bursts evolving as $n_{c}(z) \propto \exp \left(\eta \tau H_{0}\right):(a) \eta=3 ;(b) \eta=9$

additional flattening of the $\log \mathscr{N}(>P)$ distribution that offsets the increased number of bursts at larger distances.

In Figure 5, we present the maximum allowable redshift as a function of the exponential scale factor $\eta$ for three cosmological models with $\Lambda=0$ and $q_{0}=0,0.5$, and 1.0. Again, cosmological scenarios with $\Lambda=0$ and exponential density evolution that are consistent with the BATSE observation of $N(P)$ must have limiting redshifts with values less than or equal to the values of $z_{\max }$ shown in the figure. As before, the plotted points correspond to consistent scenarios with standardcandle burst populations.

Qualitatively, the curves shown in Figure 5 for exponential source evolution are similar to those shown previously for the power-law evolutionary mode, and similar conclusions to $\$ 3$ may be drawn from Figure 5.

\section{QUASAR-LIKE GAUSSIAN DENSITY EVOLUTION}

The two previous forms of PDE explored in this paper are both rather ad hoc, with little or no physical underpinning to rationalize their use as possible forms of source evolution for gamma-ray bursts. However, as mathematical functions, they offer insight into the types of effects that source evolution can have on the observational data. Both of the previous functions are monotonically increasing with redshift and/or look-back

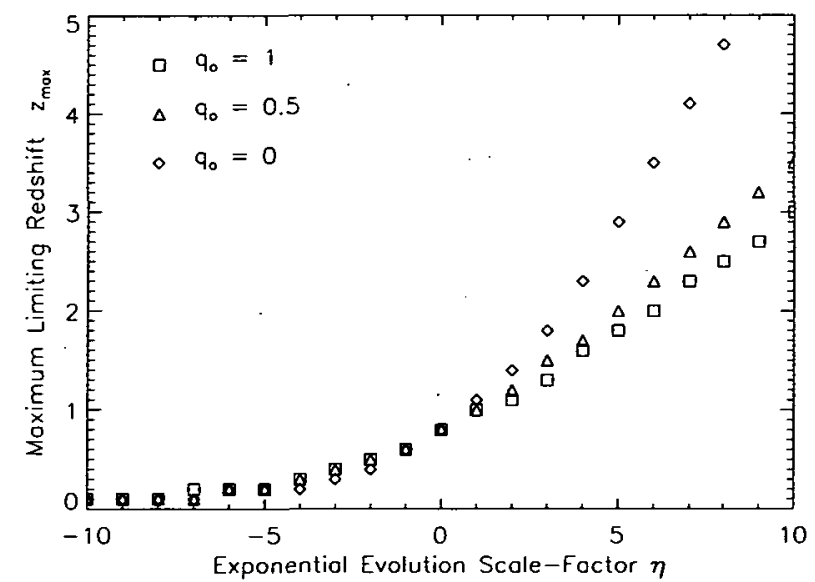

Fig. 5. - Limiting redshift $z_{\max }$ vs. exponential scale-factor $\eta$ for three $\Lambda=0$ cosmologies. time. While it is possible that over the range of observable redshifts the comoving burst rate density does continue to increase, it is reasonable to assert that at some redshift or look-back time, this comoving density of bursts may decrease to zero, certainly by the time that one reaches the bigbang. It is also possible that, as in the case with quasars, the density of sources does begin to decrease at a distance/redshift that is interior to the maximum being sampled by the instrument. A source density with a turnover interior to the volume of space sampled by the experiment will have very different consequences for the observed $N(P)$ distribution, compared to a source density that continues to increase out to the experiment's threshold.

Quasars are among the earliest visible objects to form in the universe, currently detectable to redshifts of order 5 (Schneider et al. 1989). As such, they may be the first indicators of the formation of large-scale structures, with evolutionary connections to AGNs, BL Lac objects, Seyfert galaxies, and "normal" galaxies that we observe in nearby space. Figure 6 is adapted from Hartwick \& Schade (1991) and contains the integrated space density of luminous quasars (absolute magnitude $M_{B}<$ -26) for the two parameter sets $q_{0}=0.1, \alpha_{q}=-1$ (open circles) and $q_{0}=0.5, \alpha_{q}=-0.5$ (filled circles), where $\alpha_{q}$ is the power-law spectral index of the quasars.

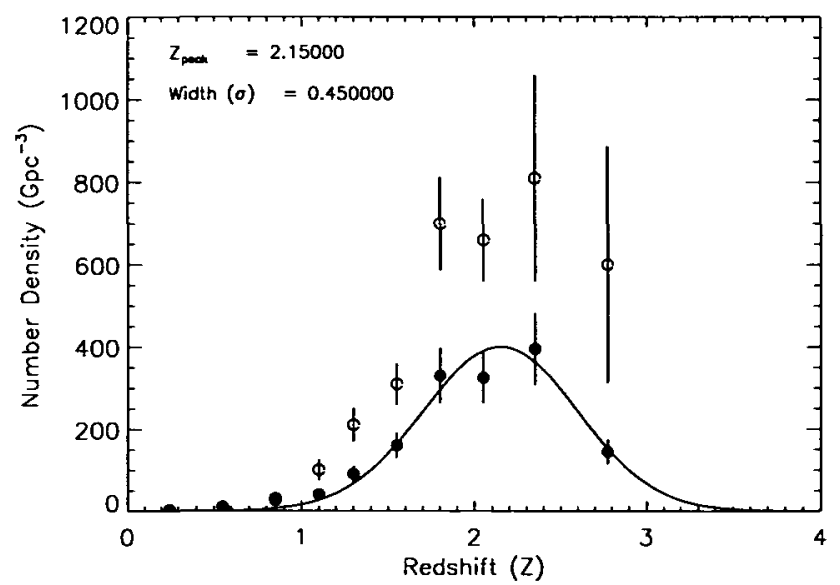

FIG. 6--Measured comoving number density of quasars for two different assumed spectral indices and cosmologies. Adapted from Hartwick \& Schade (1991). 
Although difficult to determine precisely, the comoving density of quasars increases with redshift out to a broad maximum around $z \approx 2-2.5$, followed by a general decrease in the comoving density of sources. The solid curve shown in the figure is a simple Gaussian representation of the filled-circle data, with a mathematical form

$$
n_{c}(z) \propto \exp \left[\frac{-\left(z-z_{\text {peak }}\right)^{2}}{2 \sigma^{2}}\right]
$$

with values of $z_{\text {peak }}=2.15$ and $\sigma=0.45$. This simple curve does not fit the data precisely, however it does capture the general characteristics of the source density increase, followed by a broad maximum and then a decline. Additionally, this functional form can also yield a reasonable representation of the open-circle data $\left(q_{0}=0.1, \alpha_{q}=-1\right)$ simply by adjusting the amplitude and leaving the values of $z_{\text {peak }}$ and $\sigma$ fixed.

Gamma-ray bursts at cosmological distances require the existence of some type of progenitor object which itself may follow a similar evolution in comoving density to that of the quasars. This notion may be especially relevant for objects like AGNs, which are observed to display an evolutionary connection to the quasars, and have been speculated to be one of the possible sites of gamma-ray burst production (Brainerd 1994). The redshift at which the peak density occurs, however, may be different for the gamma-ray bursts than shown in Figure 6 because of a "lag" in cosmic time between the formation of the quasars and the development of the burst progenitor objects. Similar lags occur in NS-NS merger scenarios, where binary formation and the merger event would have a large range of lag times, depending on the initial binary separation.

We therefore have chosen to explore the effects of PDE in the context of the BATSE gamma-ray burst data utilizing the comoving number density of Equation (9) with various values of $z_{\text {peak }}$ and $\sigma$. The motivation to use such a function comes from both its mathematical nature, with an actual decrease in the comoving density of sources beyond a certain redshift, as well as for its possible physical implications regarding the evolution of objects known to exist at cosmological distances.

Figure 7 displays the $z_{\max }\left(\sigma_{0}, q_{0}\right)$ surface obtained with a comoving number density prescribed by equation (9), with a value of $z_{\text {peak }}=1.0$ and a width $\sigma=0.45$. This figure is again qualitatively similar to the previous surfaces shown with other forms of density evolution. Despite the similarities, there are some interesting differences as well. The plateau region for this type of evolution appears flatter, with even less $z_{\max }$ depen-

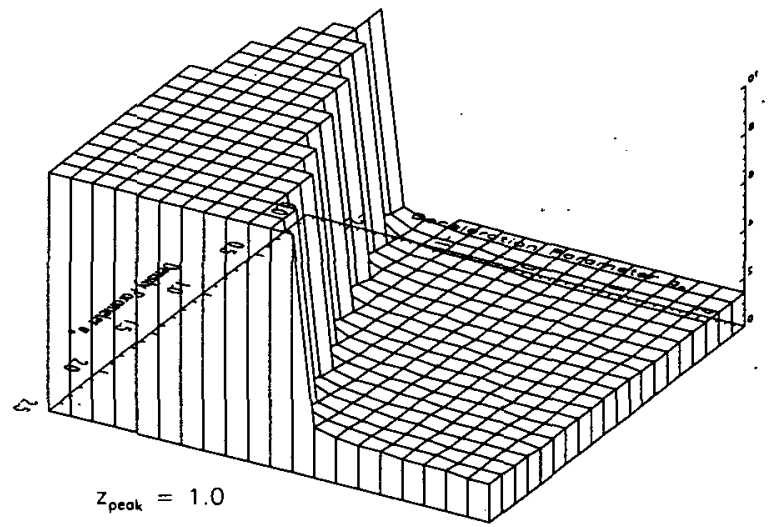

Fig. 7.--Limiting redshift $z_{\max }\left(\sigma_{0}, q_{0}\right)$ computed for a burst population which evolves according to eq. (7). For this figure, $z_{\text {peak }}=1.0$ and $\sigma=0.45$. dence on the exact values of $\sigma_{0}$ and $q_{0}$ for these cosmologies. For smaller values of $z_{\text {peak }}$, the plateau region is more extensive in area and requires smaller values of $z_{\max }$ as $z_{\text {peak }}$ is decreased. For $z_{\text {peak }}$ equal to zero (i.e., the maximum comoving density of bursts occurs locally), a maximum limiting redshift of $z_{\max } \approx$ 0.5 is found for all cosmologies with $q_{0}>-1$, regardless of the value of $\sigma_{0}$. This is because the fall-off in $n_{c}(z)$ is already sufficient to flatten the $-3 / 2$ Euclidean power law to the observed form without the need for significant cosmological effects. At large values of $z_{\text {peak }} \geq 2$, there is no obtainable constraint on $z_{\max }$, as most of the bursts are concentrated in a relatively narrow range of redshift (a "shell-model"), and an assumed luminosity function similar in form to the observed brightness distribution can always be found to make the observed spatial distribution produce a brightness distribution that is consistent with what is actually being observed by BATSE.

Figure 8 contains plots of $z_{\max }$ versus $z_{\text {peak }}$ for $\sigma=0.45$ and cosmölogies with $q_{0}=0,0.5$, and 1.0 , each with a zero cosmological constant. Both the measured value of $z_{\max }$ is shown (open symbols) as well as the $+3 \sigma$ upper limit on $z_{\max }$ (filled symbols). As with the previous two similar figures (Figs. 3 and 5), brightness distributions consistent with the BATSE data can be found for a given $z_{\text {peak }}$ and the PDE shown in equation (9) provided that a suitable luminosity function is chosen and the limiting redshift is less than or equal to $z_{\max }$. Points plotted in the figure correspond to models with nearly mono-luminous burst populations, and the required luminosity function becomes broader as the limiting redshift is decreased below the plotted value of $z_{\max }$.

Interesting conclusions can be drawn from Figure 8 with an additional assumption regarding the actual limiting redshift in the BATSE data. If we again choose the value of $z_{\text {lim }} \approx 2$, it is clear from Figure 8 that the value of $z_{\text {peak }}$ cannot be less than $\sim 1.4$ with a $3 \sigma$ lower limit of $\sim 1.0$, regardless of the value of $q_{0}$ chosen. Given the measurement of Norris et al. (1994), it is impossible for a quasar-like comoving burst density of the form of equation (9) to peak at a redshift smaller than $z_{\text {peak }} \sim$ 1.0 and still remain consistent with the observed BATSE data in $\mathbf{a} \Lambda=0$ universe.

The effect of modifying the width of the gaussian distribution $(\sigma)$ in equation $(9)$ is shown in Figure 9 where the maximum limiting redshift $z_{\max }$ is plotted as a function of $1 / \sigma$. For large values of $z_{\text {peak }}$, making the distribution narrower

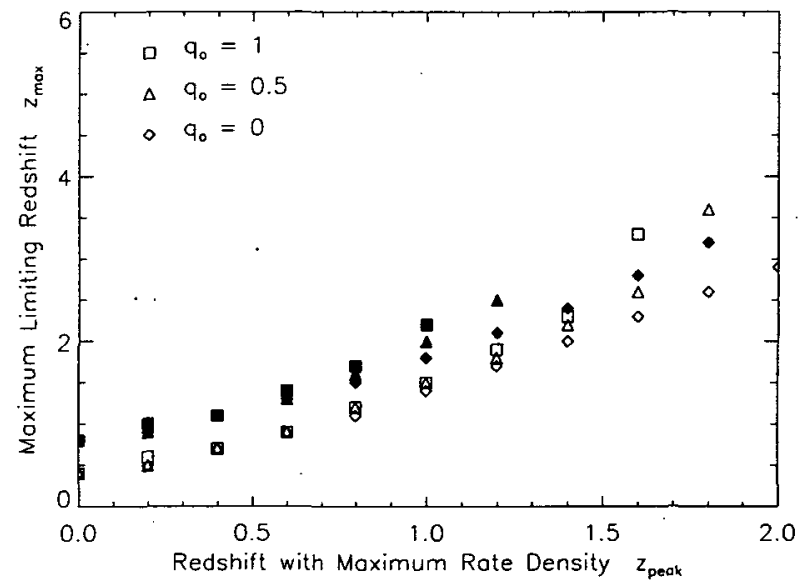

FIG. 8.-Limiting redshift $z_{\max }$ (open) and $3 \sigma$ upper limits (filled) vs. peakdensity redshift $z_{\text {pesk }}$ in three $\Lambda=0$ cosmologies with Gaussian-like evolutionary scenarios. 


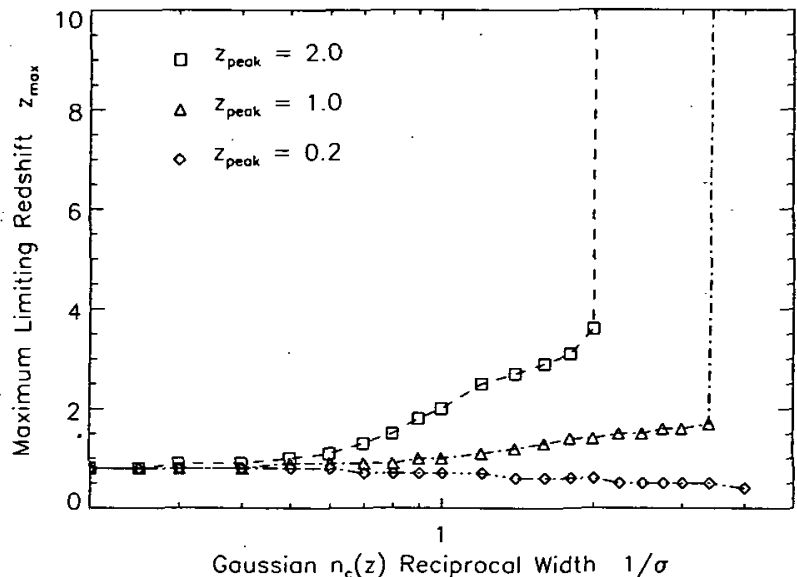

FIG. 9.--Limiting redshift $z_{\max }$ as a function of the inverse of the Gaussian $n_{c}(z)$ width $\sigma$. The computation is artificially truncated at $z_{\max }=10$.

(increasing $1 / \sigma$ ) increases the maximum limiting redshift. The narrower distribution more closely approximates the "shell model" discussed earlier, and is therefore compliant with the data over a larger range of limiting redshifts. If $z_{\text {peak }}$ is small, however, the limiting redshift is decreased as the distribution is made narrower. In this case, the steep drop-off in the burst rate density is itself sufficient to cause the observed bending in the $\log \mathscr{N}(>P)$ distribution without the introduction of significant cosmological effects that would be introduced by observing to large redshifts.

As the distribution of burst rate density is given a larger width (making $1 / \sigma$ smaller), it more closely approximates the case of a distribution that is roughly constant over a large range of redshifts, a scenario that has been widely studied in the literature (Wickramasinghe et al. 1993; Mao \& Paczyński 1992; Emslie \& Horack 1994). In agreement with these previous studies, the limiting redshifts in these cases approach $z_{\max } \sim 1$ as $\sigma$ becomes large.

If we again adopt $z_{\max }=z_{\text {lim }}=2$, as suggested by the work of Norris et al. (1995), then for monoluminous bursts we see that $z_{\text {peak }}$ must be greater than about unity, and that the width of the gaussian distribution must be at least as wide as $\sigma \approx 0.3$ depending on the exact value of $z_{\text {peak }}$. This is a rather pleasing result; such an evolutionary scenario creates an enhancement of bursts at just the right redshift, and over just about the right range of redshifts to produce the quick roll-over observed in the BATSE $\log \mathscr{N}(>P)$ distribution. This rapid roll-over has been interpreted, in a Euclidean geometry, by a width in the observed burst luminosity distribution of about a factor of 6 (Horack et al. 1994b). For $z_{\text {peak }}=1$ and $\sigma=0.33$, the spread in redshifts within which $80 \%$ of the bursts are contained is from 0.58 to 1.42 , or a range of a factor of 2.45 . The square of this quantity is indeed about 6 , the exact range of $L$-values within which $80 \%$ of the bursts must be contained if a Euclidean scenario is valid (Horack et al. 1994b).

\section{DISCUSSION}

The technique of integral moments has provided insight into three evolutionary scenarios for the comoving rate number density of gamma-ray bursts. In all three of the scenarios that have been examined, power-law evolution, exponential evolution, and a Gaussian quasar-like evolution function, many of the results are qualitatively similar. We observe for a large number of $\left(\sigma_{0}, q_{0}\right)$, including all $\Lambda=0$ models, that there exists a broad $z_{\max }\left(\sigma_{0}, q_{0}\right)$ plateau that limits the maximum possible observed redshift. As the amount of evolution is increased in a given model, the admissible value of $z_{\max }$ increases.

Introducing an externally derived value for the limiting redshift in the BATSE sample provides a means by which the amount of evolution in the burst population can be constrained. By employing the limiting redshift $z_{\text {lim }} \sim 2$ found by Norris et al. (1995) through the analysis of temporal burst profiles from the BATSE data, all mathematically tenable evolutionary scenarios with limiting redshifts that are smaller than this value for all possible luminosity functions are excluded.

A special case is the scenario in which the comoving burst density is a constant, i.e., no evolution. (See Figs. 1 and 3 with $\beta=0$, Fig. 5 with $\eta=0$, or Fig. 9 with $1 / \sigma=0$.) Very few of the nonevolving cosmological models, indeed only accelerating models where $q_{0}<0$, can have limiting redshifts of 2 , even if one chooses to use the $+3 \sigma$ upper limits to the derived $z_{\max }$ value as the quantity of interest. On the basis of this analysis and the strict application of an independent measurement of the limiting redshift in the BATSE data, we therefore conclude that if the actual limiting redshift in the BATSE data is $z_{\lim } \sim$ 2 , then there must be some form of evolution present in the gamma-ray burst population unless $\Lambda>0$. Furthermore, this evolution must be of a form to either increase the comoving density with redshift, increase the luminosity of bursts with redshift, or some combination of both.

If the evolution is $\mathrm{PDE}$, for $\Lambda=0$ cosmologies, power-law evolution must display a rate density increase at least as fast as $(1+z)^{1.5}$, and exponential evolution must increase at least as fast as $\exp \left(3 \tau H_{0}\right)$ to avoid inconsistency with the BATSE $N(P)$ distribution at some level. A gaussian-like quasar evolution function with a $\sigma \sim 0.45$ must peak at a redshift no less than $z \sim 1.0$. Employing different values of $\dot{q}_{0}$ in these $\Lambda=0$ cosmologies makes only a minor difference in these results (see Rutledge, Hui, \& Lewin 1995).

The necessity of evolution in cosmological scenarios has been discussed previously in the literature by Fenimore et al. (1992) who utilized early BATSE data in conjunction with data from PVO. However, the form of evolution that was stated to be required in their analysis was in the opposite sense to that found here, with fewer or fainter bursts at large redshifts. A re-examination of the effective PVO lifetime and subsequent analysis of the combined data (Fenimore et al. 1993) retracted the previous requirement of evolution, showing that the BATSE-PVO brightness distribution is consistent after all with a nonevolving population to limiting redshifts of order unity.

The limiting $z_{\max }\left(\sigma_{0}, q_{0}\right)$ surface derived for the three evolutionary scenarios corresponds to a mono-luminous population of gamma-ray bursts. Therefore, if one further requires that bursts possess identical luminosities in addition to the previous constraint that $z_{\max } \sim 2$ in the BATSE data, one severely restricts the amount of PDE source evolution that can be present in the data. Again for $\Lambda=0$ cosmologies, power-law type evolution of the form $n_{c}(z) \propto(1+z)^{\beta}$ is incompatible with the observational data and these assumptions outside the range $1.5<\beta<2.0$, depending somewhat on the value of $q_{0}$ chosen. In the case of exponential evolution with cosmic lookback time, the comoving density function $n_{c}(z) \propto \exp \left(\eta \tau H_{0}\right)$ is confined to the region $3<\eta<6$.

With an assumed mono-luminous burst population and an actual limiting redshift of 2 , the quasar-like Gaussian evolution function of Hartwick \& Schade (1991) shown in Figure 6, must 
peak somewhere between $1.0<z_{\text {peak }}<1.5$. Evolutionary density functions of this type which peak at redshifts smaller than $z=1$ are incompatible with the BATSE data regardless of one's assumption of the luminosity function, provided that a limiting redshift of $z=2$ is present in the BATSE $N(P)$ distribution.

In this work, we have focused only on PDE modes of evolution because of their mathematical simplicity, their possible relation to certain classes of models, and the applicability of integral moment analyses to these forms of evolving burst populations. There remains the possibility that the luminosity function of the bursts also evolves. Although the integral moment analysis technique is not equipped to handle inseparable forms of $\phi(L, z)$, a qualitative understanding of luminosity evolution and its effects on the observed $N(P)$ distribution can be understood on the basis of this analysis. Luminosity evolution which provides for more luminous bursts at large redshifts with no concurrent source evolution will require the presence of larger limiting redshifts in order that the resulting $\mathcal{N}(>P)$ distribution be consistent with the observed data. Alternatively, a decrease in burst luminosity with increasing redshift will work in the opposite direction, reducing the allowable limiting redshifts consistent with the BATSE data.
Given the freedom to invoke both luminosity and source density evolution, an extremely large number of combinations will be able to generate a theoretical brightness distribution that mimics the BATSE observed $N(P)$ distribution. However in many cases, especially those scenarios with only moderate limiting redshifts, these two evolutionary modes must work against each other to produce a resulting intensity distribution consistent with the BATSE data. Cosmological models with very strong evolution in the luminosity function that is compensated by strong evolution in the source density function (in the opposite sense) in order to produce a brightness distribution that is consistent with the BATSE data, itself consistent with a nonevolving, mono-luminous population (Wickramasinghe et al. 1993; Fenimore et al. 1993; Emslie \& Horack 1994) would be suspect through an Occam's Razor argument.

A. G. E. and D. H. H. are supported through the Compton Gamma-Ray Observatory Guest Investigator Program. We thank Jay Norris and his colleagues at the Goddard Space Flight Center for substantive discussions regarding this analysis.

\section{REFERENCES}

Brainerd, J. J. 1994, ApJ, 428, 21

Briggs, M. S., et al. 1993, in AIP Conf. Proc. 307, Gamma-Ray Bursts ed. G. J.

Fishman, K. Hurley, \& J. Brainerd (New York: AIP), 44

Emslie, A. G., \& Horack, J. M. 1994, ApJ, 435, 16

Fenimore, E. E., et al. 1992, Nature, 351, 140

. 1993, Nature, 366, 40

Fishman, G. J., et al. 1994, ApJS, 92, 229

Hartmann, D. H. 1995, in The Gamma-ray Sky with Compton GRO and SIGMA, ed. M. Signore, P. Salati, \& G. Vedrenne (Dordrecht : Kluwer), in press

Hartwick, F. D. A., \& Schade, D. 1991, ARA\&A, 28, 437

Horack, J. M. et al. 1994, ApJ, 413, 293

Horack, J. M., \& Emslie, A. G. 1994, ApJ, 428, 620

Horack, J. M., Emslie, A. G., \& Meegan, C. A. 1994b, ApJ, 426, L5

Mao, S., \& Paczyński, B. 1992, ApJ, 388, L45
Meegan, C. A., et al. 1992, Nature, 355, 143

. 1994, 2d BATSE Gamma-ray Burst Catalog, available electronically from the GRO Science Support Center

Norris, J. P., et al. 1995, ApJ, 439, 542

Pendleton, G. N., et al. 1995, in preparation

Piran, T. 1992, ApJ, 389, L45

Piran, T et al. 1991, in Proc. Huntsville GRB Workshop, ed. W. S. Paciesas \&

G. J. Fishman (New York: AIP), 149

Rutledge, R. E., Hui, L., \& Lewin, W. 1995, ApJ, submitted

Schneider, D. P., et al. 1989, AJ, 98, 1507

Wald, A. 1939, Trans. Am. Math. Soc., 46, 280

Wickramasinghe, W. A. D. T. et al. 1993, ApJ, 411, L55

Wijers, R., \& Paczyński, B. 1994, ApJ, 437, L107

Woosley, S. E. 1993, ApJ, 405, 273 